\title{
Analyzing Customer Site UI Design Experience under the Network
}

\section{Environment Development Perspective}

\author{
Shuang $\mathrm{Li}^{1, \mathrm{a}}$ \\ ${ }^{1}$ Zhengzhou University of Industrial Technology, the School of Information Engineering, Henan, 451100
}

Keywords: Network Environment; Web Site Design; User

\begin{abstract}
With the rapid development of HTML5 and CSS3 technology in the network environment, which will undoubtedly bring more fresh design to the site interactive experience? For the success of the website, to attract customers, customers can more comfortable to use and consumption is the ultimate goal. From a certain perspective, technology has brought fresh function is more important than the visual appeal, but can not exceed the technical and user experience, any kind of great technology is the ease of use for the user experience with smooth and services. UI design Web pages as part of the user experience, enabling users to produce pleasure play an important role in the visual. Easy to operate and pleasing design a web page, can attract customers prefer to visit the website to participate in the site activity and access to information websites, such as participation in social activities, and consumer behavior. When web design usability and visual aesthetics users get met, will greatly enhance the customer experience of the site.
\end{abstract}

\section{Introduction}

Customer experience of the site, the site emphasizes usability and visual experience, including features, content, form and other elements that make up the user experience, are interrelated unity. Among the many factors that affect the customer experience, the product of "availability" is a crucial element. That the availability of international standards involving easy to learn, easy to use, system effectiveness, customer satisfaction, and these factors correlate with the actual use of the environment together to evaluate specific targets. That is the design of the site from a user's perspective, architecture and design of the site, but also to see the user's needs, to meet customer needs. Website design is among the user into the design, the access to information websites, consumer products as a process for a better experience.

\section{The Customer Site Experience}

Site experience. The site can be divided into three areas according to the final destination site design: website look, user behavior, the intensity of services. The user experience is the product of a progressive, first, how the visual experience when the user enters the site, there is a general impression that basis, if the first impression your site design looks UI can not meet user expectations, are likely to continue to give up visit the website. Followed by behavior, that is, website usability and ease of use, how to use the feeling, if resistance is encountered in the course of a large impact in some way would interactions. Finally, the intensity of services, when a user uses a web site especially electricity supplier site, is mainly produced consumer behavior, site of after-sales service is extremely important in the user experience, service quality directly affects the user's psychological feelings.

Customer behavior. Different types of sites, audiences will be different, such as men, women, 
teenagers and children. Determine the user groups according to their site targeting, such as automotive Web site users are mainly male-dominated. Psychology from the user's perspective, the first design of the site is the ability to focus on the user's website to promote the products, the information, you can follow their subjective wishes, does not require much thought process, you do not need to understand the site structure, icon meaning, and where navigation. Second, users do not have to spend too much learning costs, operation and understanding with fewer failures, easy-action and operating practices are good basic experience. For the experience of a website in terms of the best way is the way to design in accordance with the user in mind.

Designer elements. For according to the work site may have content into visual designers, interaction designers and process engineering designers. Visual designers need to consider is whether the creative web design and meet the aesthetic needs of the user, the user experience site looks directly generated from the appearance. Interaction designer is responsible for interactions between users and websites, in this process, the user can smoothly complete the process of experience. Program engineers decided to functional and technical support Web site, the site is able to ensure the normal operation, it does not affect the user.

\section{The Relationship between UI Design and Experience}

That UI user interface, is a medium of communication and information exchanged between the system and the user, it implements internal information in the form of the human form can accept the conversion between. For the field of industrial design in HCI design refers to the time the interaction between humans and machines can be efficient, and comfortable handling of the machine, the operator is required to make a decision operation of the machine while the machine can also help quickly feedback to the user.

UI design Web pages. Site UI design embodied in the design of the user interaction with the web site between. From the perspective of the user interface, the web UI design is closely related to the page visuals, style, not only has aesthetic value, but also embodies the humane care, in terms of people's reading habits or operating behavior have people-oriented concept. Of course, for a site, the user experience is not just aesthetic and functional design. It usually involves coordination function and form the most balanced, as the goal of the entire site and service, which will narrow the distance between the user and the website, pleasing appearance and powerful function is fit properly, become a test user experience users to easily use the site monthly, the higher the satisfaction, the more smooth interaction.

Reasonable page layout helps the user to browse, understand pages of information, especially for such as So-tho, Sienna and other portals is critical, how that enables the user to find a need in many information. Making web design, to content-based, rational distribution of information in accordance with the block, do the emphasis and detail of process.

Web page style establishment visual information is the key to the transition to the information in the form of visual designers based on the content of the site generated, expressed through colors, images, text and other visual elements. E-commerce sites such as the pursuit of more information and number sense, good-looking design may not be the actual data more attractive. This site features relatively wide, such as Dan-dong, TaoBao, product classification clearly reflects the potential user groups, can more effectively interact to achieve consumption.

The appearance of the site is mainly reflected in the visual elements on a page, users will have a relation of color pages, graphics, text between a certain psychological impact of such changes affect not only embodies the elements of the position, size in, and reflected in the the actual situation on spatial relationship. Color as one element of human emotional expression, often the first impression a user 
browsing the site. Graphics can achieve a good effect to convey and guide the user access to information process. Text as a means to convey information on the main website, has a role can not replace, either to or from the title page text content, can do detailed and accurate delivery.

Web design aesthetics and user experience closely, UI design is necessary to design the visual effects in the user's point of view, but also to study the user's habit of thinking in web design directly reflected in the colors, layout, graphics, text them, guide and influence user access to information. Web design is not just gorgeous pages do, or to allow people to be able to interface visual elements to guide and cater to the user through the operation, rather than just the pursuit of creating visual effects. Fundamentally speaking, a good web design should have a clear presentation of information, can be a good guide the user to complete the task site. When designing a user interface when we must take into account the suitability of the user interface, it needs to consider the user's age, lifestyle, and ultimately to the user's design, which is a rational design process. So to UI design and user research closely, take the initiative to consider the feelings and needs of the user.

\section{Conclusion:}

Thus, in the digital age with a user-centered website design user experience will become increasingly important, in terms of usability for the user or site UI design will deeply affect the user's actions and experience. It is an important factor affecting the readability of web page design aesthetic value of UI design. Rational UI design will make the user a pleasant experience to enjoy the visual design of the page, while the whole process is bound to increase the attractiveness of the site, prompting the user to read, to spend. A scattered layout, styles affect the user interface to click and consumer desire. Core user-concentric UI design Website Design, which is a variety of platforms product design trend in the future.

\section{References:}

[1] UI Evolution: mobile human-computer interface design Author: Fuzhou Than Press: Songhai University Press Publication Date: January 1, 2010

[2] Seven days proficient Photo shop CS5 UI interaction design Author: Chang Xiaoping edited Publisher: Electronic Industry Press Publication date: 2012-1-1

[3] Ling Ruiz. Website development stages of education [J]. Theorists. 2005 (08)

[4] Fang Hafnium. Web design of small education [J]. Journal of Ujungpandang College of Education. 2005 (06)

[5] Li Weenie. Basic elements of education website construction [J]. Michelangelo education (consolidated version). 2004 (16) 